\title{
Host genetic regulation of immune-based and infectious diseases
}

\author{
Introduction to mammalian genome special issue: genetics of infectious disease
}

\author{
Martin T. Ferris ${ }^{1} \cdot$ Derek W. Hood ${ }^{2}$
}

Published online: 31 August 2018

○) Springer Science+Business Media, LLC, part of Springer Nature 2018

Infectious disease remains a leading cause of mortality worldwide and includes a wide range of ailments caused by a pathogen, typically a virus, bacterium, fungus or parasite, entering and living in the host body. For many decades, but particularly after the molecular biology revolution brought about by the advent of recombinant DNA techniques in the 1970s, the tenet for an infectious disease biologist was to identify genes on the microbe that might play a role in the pathogenic traits of that organism. By first cloning that gene, mutating it in a defined way, analysing the altered phenotype or function of the mutant strain then restoring the gene function to re-establish pathogenicity and satisfy Koch's postulates, it was assumed this would lead to a complete understanding of disease. Koch's postulates however imply that virulence traits reside solely in the microbe and are therefore independent of the host. It is now clear that this aspect of the postulate is erroneous and that indeed the susceptibility of the host is paramount and typically as important, if not more important, than the traits of the microbe in determining the outcome of infection and disease.

Indeed, with advances in genetics concurrent with those described above for molecular biology, it was clear that host genetic contributions were a major contributor to infectious disease outcomes, along with a wide range of environmental and demographic variables (e.g. climate, sex, age, immune history). Together these complex factors and their interactions were understood, along with pathogen differences, to lead to the wide spectrum of disease outcomes across individual hosts. Indeed, for human infectious disease, genetic diversity is a key determinant modulating its outcome.

Martin T. Ferris

mtferris@email.unc.edu

1 Department of Genetics, University of North Carolina, Chapel Hill, NC 27599, USA

2 Mammalian Genetics Unit, MRC Harwell Institute, Harwell Campus, Oxfordshire OX11 0RD, UK
Despite this awareness, it was still technically and ethically challenging to understand how and which human genetic variants could impact disease outcomes. Cell biology offered one means to investigate and understand how microbes exploit normal host cell mechanisms for their own end but this by no means provides the insight into the multitissue/whole-organism processes necessary to understand disease in a multicellular host expressing the complex layers and interacting components of an immune system. Indeed, such early approaches highlighted the genetic complexity of the immune system and responses to infectious diseases. As the study of infectious disease is by definition complex, sophisticated experimental investigation is crucial to allow us to gain an understanding that can improve human health. Model infection systems in animals therefore offer a critical means to explore and assess the combined effect of the plethora of pathogen-host interactions that cumulatively determine the outcome of infection. These systems not only allow us to gain mechanistic insights into the processes that initiate and progress disease but can also provide an invaluable means to develop and assess novel therapeutics. Indeed, even as advances in human genetics have allowed for larger population-based genetic analysis of chronic disease, and other biomedically important traits via non-interventionist approaches, experimental models of infectious diseases have been maintained as the golden standard in infectious disease research, based on the ability to prove disease causality via intervention and manipulation.

This special issue of Mammalian Genome comes at a time of our rapidly advancing knowledge of infectious disease and provides major review and updates for studies in the natural host and improved model infection systems for the four major pathogen types, virus, bacterium, fungus and protozoan. The development of new experimental approaches has facilitated significant advance in our understanding of the complex host-microbial interactions that contribute to disease, in particular from the perspective of 
the host. Articles included in this issue describe studies on host interaction and pathogenesis of important viral pathogens: Coronavirus (Cockrell et al.), Flavivirus (Manet et al.), Alphavirus (Baxter and Heise), Herpes simplex (Mancini and Vidal) and Influenza (Kollmus et al.). The latter study utilises the collaborative cross (CC) mouse, enabling the major factor of host genetic variation and susceptibility to be better factored into infection studies, the $\mathrm{CC}$ mouse is also utilised by Atamni et al. to study organisms from a number of the pathogen categories. Studies of the protozoan Plasmodium and malaria in mouse models are described and reviewed in the articles by Torre et al. and Huang et al. Host susceptibility and pathogenesis in animal models and the natural host are described for significant bacterial pathogens: Mycobacterium tuberculosis in man (Dallman-Sauer et al.) and Mycobacterium avium in cattle (Kiser et al.), Pseudomonas aeruginosa (Loré et al.) and Enterobacteria (Kang et al.). Due to the propensity for utilising mouse models to study infectious disease, the immunological traits of wildderived mice are compared to inbred mice (Poltorak et al.) and loci determining sensitivity to sepsis are investigated and discussed (Timmermans et al.). Interferon-stimulated genes dependent upon viral infection (Green et al.) and the immunological deficiencies leading to hyper-IgE syndrome in human and mouse models (Zhang et al.) are discussed and reviewed.

The collective contribution of the articles in this Special Issue of Mammalian Genome is to provide a significant updated overview of our knowledge of the genetics of infectious disease through studies in the host and animal models. Continued investigations whereby we understand the primary role that the host plays in determining interactions with the pathogen that lead to disease, or not, should enable improved strategies to treat and prevent this major cause of morbidity and mortality in the human population. 